PROCEEDINGS OF THE

AMERICAN MATHEMATICAL SOCIETY

Volume 128, Number 1, Pages 111-118

$\mathrm{S}$ 0002-9939(99)05117-5

Article electronically published on March 3, 1999

\title{
AN EXTENSION OF A NON-COMMUTATIVE CHOQUET-DENY THEOREM
}

\author{
G. A. WILLIS
}

(Communicated by Palle E. T. Jorgensen)

\begin{abstract}
Let $G$ be a discrete group, and let $N$ be a normal subgroup of $G$. Then the quotient map $G \rightarrow G / N$ induces a group algebra homomorphism $T_{N}: \ell^{1}(G) \rightarrow \ell^{1}(G / N)$. It is shown that the kernel of this map may be decomposed as $\operatorname{ker}\left(T_{N}\right)=R+L$, where $R$ is a closed right ideal with a bounded left approximate identity and $L$ is a closed left ideal with a bounded right approximate identity. It follows from this fact that, if $I$ is a closed twosided ideal in $\ell^{1}(G)$, then $T_{N}(I)$ is closed in $\ell^{1}(G / N)$. This answers a question of Reiter.
\end{abstract}

The main result of this paper is an extension of a theorem from [W1]. As is explained below, that theorem may be regarded as a non-commutative ChoquetDeny theorem. The extension has implications for the structure of group algebras and, in particular, answers a question of Reiter [Re1], 8.4.6. This paper is essentially the one announced in the notes added in proof to [W1], although it is not exactly what was intended then.

Throughout, $G$ will denote a locally compact group and $L^{1}(G)$ and $M(G)$ the usual group and measure algebras with convolution product. The Haar measure on $G$ will be denoted by $m ; \mu$ will denote an arbitrary probability measure on $G$. A probability measure which is absolutely continuous with respect to $m$ is said to be non-degenerate if the smallest closed subsemigroup supporting $\mu$ is $G$ itself. We shall identify $L^{1}(G)$ with the ideal in $M(G)$ consisting of all measures which are absolutely continuous with respect to $m$, so that $\mu * f$ and $f * \mu$ are well-defined whenever $f$ belongs to $L^{1}(G)$; see [H-R], Definition (20.5).

Let $\mu$ be a probability measure. Define

$$
{ }_{\mu} J=\left\{\mu * f-f: f \in L^{1}(G)\right\}^{-}, \quad J_{\mu}=\left\{f * \mu-f: f \in L^{1}(G)\right\}^{-}
$$

and

$$
L_{0}^{1}(G)=\left\{f \in L^{1}(G): \int_{G} f d m=0\right\} .
$$

Then $L_{0}^{1}(G)$ is a two-sided ideal. Also, ${ }_{\mu} J$ is a right ideal with a bounded left approximate identity, $J_{\mu}$ is a left ideal with a bounded right approximate identity and both of these ideals are contained in $L_{0}^{1}(G)$.

The Choquet-Deny theorem asserts that, if $G$ is abelian and if $G$ itself is the smallest closed subgroup supporting $\mu$, then $L_{0}^{1}(G)=J_{\mu}$; see [C-D]. Of course, since

Received by the editors June 24, 1995 and, in revised form, September 5, 1995 and March 10, 1998.

1991 Mathematics Subject Classification. Primary 43A20; Secondary 22D40. 
$G$ is abelian, ${ }_{\mu} J=J_{\mu}$ in this case. It cannot be true in general that $L_{0}^{1}(G)=J_{\mu}$ because $J_{\mu}$ has a bounded right approximate identity but $L_{0}^{1}(G)$ does not have a bounded right approximate identity unless $G$ is amenable; see [Re2]. In [W1], the following non-commutative version of the Choquet-Deny theorem is proved:

Theorem 1. Let $\mu$ be a non-degenerate probability measure on $G$. Then

$$
L_{0}^{1}(G)={ }_{\mu} J+J_{\mu} .
$$

(A new and clearer proof of this theorem is sketched below.)

The present paper concerns relativisations of Theorem 1, in the sense defined in [Re1]. Let $N$ be a closed, normal subgroup of $G$ and denote by $T_{N}: L^{1}(G) \rightarrow$ $L^{1}(G / N)$ the group algebra homomorphism induced by the quotient map $G \rightarrow$ $G / N$. Note that, if $N=G$, then $\operatorname{ker}\left(T_{N}\right)=L_{0}^{1}(G)$. Also, when $\mu$ is supported in $N, J_{\mu}$ and ${ }_{\mu} J$ are contained in $\operatorname{ker}\left(T_{N}\right)$.

We can therefore hope to relativise Theorem 1 by proving, for each normal subgroup $N$, any of the following, successively weaker, statements:

(1) for each pair, $\mu_{1}$ and $\mu_{2}$, of non-degenerate probability measures on $N$,

$$
\operatorname{ker}\left(T_{N}\right)={ }_{\mu_{1}} J+J_{\mu_{2}} ;
$$

(2) for each non-degenerate probability measure, $\mu$, on $N$,

$$
\operatorname{ker}\left(T_{N}\right)={ }_{\mu} J+J_{\mu} ;
$$

(3) there is a probability measure, $\mu$, on $N$ such that

$$
\operatorname{ker}\left(T_{N}\right)={ }_{\mu} J+J_{\mu} ; \text { and }
$$

(4) there are probability measures, $\mu_{1}$ and $\mu_{2}$, on $N$ such that

$$
\operatorname{ker}\left(T_{N}\right)={ }_{\mu_{1}} J+J_{\mu_{2}} .
$$

Of these statements, (2) would be the most direct generalization of Theorem 1. However, (2) would follow once (1) could be proved in the case $N=G$. As is discussed in [W1], the relativisation of Theorem 1 is relevant to some factoring problems in certain ideals in $L^{1}(G)$. Indeed, if (4) holds, then $\operatorname{ker}\left(T_{N}\right)$ is the sum of a closed, right ideal with a bounded left approximate identity and a closed, left ideal with a bounded right approximate identity.

In $[\mathrm{K}], \mathrm{V}$. A. Kaimanovich studied the condition

$$
L_{0}^{1}(G)={ }_{\mu_{1}} J+J_{\mu_{2}},
$$

where $\mu_{1}$ and $\mu_{2}$ are probability measures on $G$. If this condition is satisfied, he calls the pair $\left(\mu_{1}, \mu_{2}\right)$ of probability measures a Liouville pair. In response to a question of the author, he shows that there are non-degenerate probability measures, $\mu_{1}$ and $\mu_{2}$, on free groups such that $\left(\mu_{1}, \mu_{2}\right)$ is not a Liouville pair; see Corollary 2 to Theorem 6 in $[\mathrm{K}]$. Thus (1) is false even in the case when $N=G$. These examples may be used to show that (2) is false also. It is possible that similar methods will show that (3) too is false. However, it is shown in this paper that (4) is true in great generality, in particular in the case when $G$ is discrete. The proof relies on some results from [W1]. 


\section{SOME LEMMAS}

The first result required is Theorem 3.8 from [W1]. (There is a misprint in the statement there, the subscript 0 is missing from $L_{0}^{1}(G)$.)

Theorem 1. Let $\mu$ be a non-degenerate probability measure on $G$. Then

$$
L_{0}^{1}(G)={ }_{\mu} J+J_{\mu} .
$$

A sketch of a new proof of this theorem follows. It is shorter and clearer than that in [W1]. It also avoids some technical details which are given in [W1] but are not important in the present context. Another proof of this theorem has been given in $[\mathrm{K}]$.

Sketch of proof. The result does not require $G$ to be a group, merely a semigroup. In fact it suffices to prove the result for 'measured free semigroups' and then to pass to the general (semi)group case by quotienting.

Let $(X, \mu)$ be a probability space, and let $L^{1}(X, \mu)$ be the usual Lebesgue space. For each positive integer $n$ let $L_{n}$ be the space $L^{1}\left(X^{n}, \mu^{n}\right)$. Let $\mathcal{A}$ be the Banach space $\mathcal{A}=\left(\bigoplus_{n=0}^{\infty} L_{n}\right)_{\ell^{1}}$ and denote elements of $\mathcal{A}$ by $\mathbf{f}=\left(f_{0}, f_{1}, f_{2}, f_{3}, \ldots\right)$, where $f_{n}$ belongs to $L_{n}$. (When $n=0$ put $L_{n}=\mathbb{C}$.) Then the maps from $L_{m} \times L_{n} \rightarrow$ $L_{m+n}:\left(f_{m}, g_{n}\right) \mapsto f_{m} \otimes g_{n}(m, n=0,1,2,3, \ldots)$ extend in a unique way to a Banach algebra product on $\mathcal{A}$. This algebra is the $L^{1}$-algebra of the 'measured free semigroup with unit' generated by $(X, \mu)$. Define a map $\phi: \mathcal{A} \rightarrow \mathbb{C}$ by $\phi(\mathbf{f})=\sum_{n=0}^{\infty}\left(\int_{X^{n}} f_{n} d \mu^{n}\right)$. Then $\phi$ is a multiplicative linear functional and so $\mathcal{A}_{0}=\operatorname{ker}(\phi)$ is a closed, two-sided, codimension one ideal in $\mathcal{A}$.

Put $\mathbf{u}=\left(0,1_{X}, 0,0, \ldots\right)$, where $1_{X}$ is the constant function with value 1 and define $J=\{\mathbf{f}-\mathbf{f u}: \mathbf{f} \in \mathcal{A}\}^{-}$. Then $J$ is a closed, left ideal in $\mathcal{A}$ with a bounded right approximate identity. Since $\phi(\mathbf{u})=1, J$ is contained in $\mathcal{A}_{0}$. A key step in the proof is to show that the quotient module $\mathcal{A} / J$ is isometrically isomorphic to $L^{1}\left(X^{\infty}, \mu^{\infty}\right)$. The isomorphism is also an isomorphism of left $\mathcal{A}$-modules when the $\mathcal{A}$-module action on $L^{1}\left(X^{\infty}, \mu^{\infty}\right)$ is defined, for $f_{m}$ in $L_{m}$ and $F$ in $L^{1}\left(X^{\infty}, \mu^{\infty}\right)$, by

$$
\left(f_{m} . F\right)\left(x_{1}, x_{2}, x_{3}, \ldots\right)=f_{m}\left(x_{1}, x_{2}, \ldots, x_{m}\right) F\left(x_{m+1}, x_{m+2}, \ldots\right) .
$$

Now $\mathcal{A}_{0} / J$ is mapped under the isomorphism to $L_{0}^{1}\left(X^{\infty}, \mu^{\infty}\right)$ and it may be shown, by using the ergodic theorem for operators as in Theorem 3.8 from [W1], that $L_{0}^{1}\left(X^{\infty}, \mu^{\infty}\right)=\left[(1-\mathbf{u}) \cdot L^{1}\left(X^{\infty}, \mu^{\infty}\right)\right]^{-}$. Hence, by pulling back to $\mathcal{A}$, we have that

$$
\mathcal{A}_{0}=K+J
$$

where $K=\{\mathbf{f}-\mathbf{u f} \in \mathcal{A}\}^{-}$. This completes the proof for the semigroup algebra $\mathcal{A}$.

The result for group algebras may now be deduced. Since $\mu$ is non-degenerate, it may be supposed that it is equivalent to the Haar measure on $G$-replace $\mu$ by a convex combination of its powers if necessary. If $G$ is discrete, there is a natural Banach algebra homomorphism from $\mathcal{A}$ to $L^{1}(G)$ such that $\mathbf{u}$ is mapped to $\mu$. This homomorphism is surjective, maps $\mathcal{A}_{0}$ to $L_{0}^{1}(G), K$ to ${ }_{\mu} J$ and $J$ to $J_{\mu}$. The result follows in this case. For $G$ non-discrete, the range of the natural Banach algebra homomorphism is $L^{1}(G)$ with unit adjoined and a further approximate identity argument is needed to reach the desired conclusion.

The second result required is Lemma 1.1 from [W1]. Although the statement given here is more general than that given in [W1], the proof is identical. 
Theorem 2. Let $X$ be a Banach space, let $\mathcal{F}$ be a norm-closed, convex semigroup of contraction operators on $X$, and let $Y$ be a separable subspace of $X$ such that:

(i) $(I-T) X \subset Y$ for every $T$ in $\mathcal{F}$; and

(ii) for each $\varepsilon>0$ and each $y$ in $Y$ there is $T$ in $\mathcal{F}$ with $\|T y\|<\varepsilon$.

Then there is $T$ in $\mathcal{F}$ such that $Y=[(I-T) X]^{-}$.

This theorem may be thought of as being a variant of Cohen's factorisation theorem. It says that, if $Y$ can be approximated from below by subspaces of the form $[(I-T) X]^{-}$, then $Y$ itself has this form.

\section{The Relative Choquet-Deny theorem}

We are now ready to prove a relative version of Theorem 1 .

Theorem 3. Let $G$ be a countable, discrete group, let $N$ be a normal subgroup of $G$, and let $\mu$ be a non-degenerate probability measure on $N$. Then there is a probability measure $\hat{\mu}$ on $N$ such that

$$
\operatorname{ker}\left(T_{N}\right)={ }_{\hat{\mu}} J+J_{\mu} .
$$

Proof. Put $X=L^{1}(G) / J_{\mu}$ and $Y=\operatorname{ker}\left(T_{N}\right) / J_{\mu}$. Since $G$ is countable, $X$ is a separable space. For each probability measure $\nu$ on $N$, let $T_{\nu}$ be the operator on $X$ induced by the convolution operator $f \mapsto \nu * f$ on $L^{1}(G)$. Since $\nu$ is supported on $N,\left(I-T_{\nu}\right) X \subset Y$. Also, $\mathcal{F}=\left\{T_{\nu}: \nu\right.$ a probability measure on $\left.N\right\}$ is a norm closed, convex semigroup of operators on $X$. Hence all of the conditions for Theorem 2 are satisfied except perhaps for (ii).

To complete the proof it will suffice to show that (ii) is satisfied because then Theorem 2 will imply that there is an operator $T_{\hat{\mu}}$ in $\mathcal{F}$ such that $\operatorname{ker}\left(T_{N}\right) / J_{\mu}=$ $\left[\left(I-T_{\hat{\mu}}\right) X\right]^{-}$. Pulling back to $L^{1}(G)$ then yields

$$
\operatorname{ker}\left(T_{N}\right)={ }_{\mu} J+J_{\mu}
$$

as required.

Let $f+J_{\mu}$ be in $Y$. Since it suffices to check (ii) on a dense subspace of $Y$, it may be supposed that $f$ has finite support in $G$. Then $f$ is supported on finitely many cosets, $x_{1} N, x_{2} N, \ldots, x_{n} N$ of $N$, and so we have that $f=\sum_{k=1}^{n} x_{k} * f_{k}$, where $f_{k}$ belongs to $L_{0}^{1}(N)$ for each $k$ and, with an abuse of notation, $x_{k}$ denotes the point mass at $x_{k}$ in $G$.

By replacing $\mu$ with a convex combination of its powers if necessary, it may be supposed that $\operatorname{supp}(\mu)=N$. Then it follows from Theorem 1 that, for each $g$ in $L_{0}^{1}(N),\left\|\mu^{m} g \mu^{m}\right\| \rightarrow 0$ as $m \rightarrow \infty$. In particular, given $\varepsilon>0$, there is an $m_{1}$ such that $\left\|\mu^{m_{1}} f_{1} \mu^{m_{1}}\right\|<\varepsilon / n$. Put $\nu_{1}=x_{1} * \mu^{m_{1}} * x_{1}^{-1}$. Then $\left\|\nu_{1} *\left(x_{1} * f_{1}\right) * \mu^{m_{1}}\right\|<\varepsilon / n$ and, since $N$ is a normal subgroup, $\nu_{1}$ is supported on $N$. Next, $x_{2}^{-1} * \nu_{1} * x_{2} * f_{2}$ belongs to $L_{0}^{1}(N)$ and so there is an $m_{2}$ such that $\left\|\mu^{m_{2}} *\left(x_{2}^{-1} * \nu_{1} * x_{2} * f_{2}\right) * \mu^{m_{2}}\right\|<$ $\varepsilon / n$. Put $\nu_{2}=x_{2} * \mu^{m_{2}} * x_{2}^{-1} * \nu_{1}$. Then $\left\|\nu_{2} *\left(x_{2} * f_{2}\right) * \mu^{m_{2}}\right\|<\varepsilon / n$ and $\nu_{2}$ is supported on $N$. Also, since $x_{2} * \mu^{m_{2}} * x_{2}^{-1}$ is a probability measure, $\left\|\nu_{2} *\left(x_{1} * f_{1}\right) \mu^{m_{1}}\right\|<\varepsilon / n$. Repeating this argument for each of the cosets $x_{1} N, x_{2} N, \ldots, x_{n} N$ in turn, we find integers $m_{1}, m_{2}, \ldots, m_{n}$ such that, putting

$$
\nu=\nu_{n}=\left(x_{n} * \mu^{m_{n}} * x_{n}^{-1}\right) * \cdots *\left(x_{2} * \mu^{m_{2}} * x_{2}^{-1}\right) *\left(x_{1} * \mu^{m_{1}} * x_{1}^{-1}\right),
$$

we have that $\left\|\nu * x_{k} * f_{k} * \mu^{m_{k}}\right\|<\varepsilon / n$ for each $k=1,2, \ldots, n$. It follows that $\left\|\nu * f+J_{\mu}\right\|<\varepsilon$ and so (ii) is satisfied. 
A difficulty arises if it is attempted to extend the above proof to all locally compact groups because it is no longer possible to reduce the norm of $f$ coset by coset.

One way to attempt to avoid this difficulty is to use structure theory for locally compact groups. The next theorem deals with the connected case. For this, note that each locally compact group, $G$, has a greatest amenable, normal subgroup $N$. If $G$ is also separable or compactly generated, then, by [W1], Proposition 5.5, there are non-degenerate probability measures, $\mu$, on $G$ with $\operatorname{ker}\left(T_{N}\right) \subset J_{\mu}$.

Theorem 4. Let $G$ be a connected locally compact group, and let $N$ be its greatest closed, normal, amenable subgroup. Let $\mu_{1}$ and $\mu_{2}$ be non-degenerate probability measures on $G$ such that $\operatorname{ker}\left(T_{N}\right) \subset J_{\mu_{1}}$ and $\operatorname{ker}\left(T_{N}\right) \subset J_{\mu_{2}}$. Then

$$
L_{0}^{1}(G)={ }_{\mu_{1}} J+J_{\mu_{2}} .
$$

Proof. As shown in [M-Z], $G$ has a compact, normal subgroup, $K$, such that $G / K$ is a Lie group. Since compact groups are amenable, it follows that $G / N$ is a connected Lie group. Since solvable groups are amenable, it follows that $G / N$ is semisimple. Hence we may suppose that $G$ is a connected, semisimple, Lie group.

If $\mu$ is any non-degenerate probability measure on a connected, semisimple Lie group, then $L^{1}(G) / J_{\mu}$ is isomorphic to $L^{1}(B(T), \nu)$, where $B(G)$ is the maximal boundary defined by Furstenberg and $\nu$ is a quasi-invariant measure on $B(G)$; see [F], Theorem 5.3 and [W1], Section 2. Since $G$ acts transitively on $B(G)$, all quasi-invariant measures on $B(G)$ are equivalent. Hence the left $L^{1}(G)$-modules $L^{1}(G) / J_{\mu_{1}}$ and $L^{1}(G) / J_{\mu_{2}}$ are isomorphic.

Theorem 1 shows that the closure of $\left(\delta_{e}-\mu_{1}\right) * L^{1}(G) / J_{\mu_{1}}$ has codimension one in $L^{1}(G) / J_{\mu_{1}}$. Since $L^{1}(G) / J_{\mu_{1}}$ and $L^{1}(G) / J_{\mu_{2}}$ are isomorphic $L^{1}(G)$-modules, it follows that the closure of $\left(\delta_{e}-\mu_{1}\right) * L^{1}(G) / J_{\mu_{2}}$ has codimension one in $L^{1}(G) / J_{\mu_{2}}$. The assertion of the theorem follows.

Corollary 1. Let $G$ be a locally compact group, and let $N$ be a connected, closed, normal subgroup. Then there is a non-degenerate probability measure on $N$ such that

$$
\operatorname{ker}\left(T_{N}\right)={ }_{\mu} J+J_{\mu} .
$$

Proof. Let $f$ be in $\operatorname{ker}\left(T_{N}\right)$. Then, for almost every $x$ in $G, \int_{N} f(x y) d m_{N}(y)=0$. Hence, by the theorem, $\int_{N}\left|\left(\mu^{n} * f * \mu^{n}\right)(x y)\right| d m_{N}(y) \rightarrow 0$ as $n \rightarrow \infty$ for almost every $x$ in $G$. Therefore

$$
\begin{aligned}
\left\|\mu^{n} * f * \mu^{n}\right\|_{1} & =\int_{G}\left|\left(\mu^{n} * f * \mu^{n}\right)(x)\right| d m_{G}(x) \\
& =\int_{G / N} \int_{N}\left|\left(\mu^{n} * f * \mu^{n}\right)(x y)\right| d m_{N}(y) d m_{G / N}(\dot{x}) \\
& \rightarrow 0 \text { as } n \rightarrow \infty
\end{aligned}
$$

by the dominated convergence theorem.

The remaining difficulty with proving Theorem 3 for arbitrary locally compact groups therefore is the totally disconnected case. This case can perhaps be dealt with by using techniques from [W2]. 


\section{THE STRUCTURE OF GROUP ALGEBRAS}

The decomposition of $\operatorname{ker}\left(T_{N}\right)$ given in Theorem 3 answers some open questions about the structure of group algebras. The first question concerns factoring in various ideals in $L^{1}(G)$. It follows from Theorem 3 and the factorisation theorem of Cohen that, if $G$ is discrete and $N$ is a normal subgroup of $G$, then each element of $\operatorname{ker}\left(T_{N}\right)$ is a sum of two products. (Each element of $\ell^{1}(G)$ is supported on a countable subgroup of $G$ and so the theorem may be applied to arbitrary groups.) A similar conclusion may be drawn about some other ideals.

Theorem 5. Let $G$ be a discrete group, and let $I$ be a closed ideal in $\ell^{1}(G)$ such that $\ell^{1}(G) / I$ is finite dimensional and commutative. Then each element of $I$ is a sum of three products of elements of $I$.

Proof. Put $N$ equal to the commutator subgroup of $G$. Then $N$ is a normal subgroup of $G, \ell^{1}(G) / \operatorname{ker}\left(T_{N}\right) \simeq \ell^{1}(G / N)$, which is commutative, and $\operatorname{ker}\left(T_{N}\right) \subset I$. It follows that $I / \operatorname{ker}\left(T_{N}\right)$ is isomorphic to a finite codimensional ideal in the commutative group algebra $\ell^{1}(G / N)$ and hence that $I / \operatorname{ker}\left(T_{N}\right)$ has a bounded approximate identity.

Let $f$ be in $I$. Then, since $I / \operatorname{ker}\left(T_{N}\right)$ has a bounded approximate identity, there are $g_{1}$ and $g_{2}$ in $I$ such that $f-g_{1} * g_{2}$ is in $\operatorname{ker}\left(T_{N}\right)$. It follows from Theorem 3 that there are $a_{i}$ and $b_{i}$ in $\operatorname{ker}\left(T_{N}\right)$ such that $f-g_{1} * g_{2}=a_{1} * a_{2}+b_{1} * b_{2}$. Therefore $f$ is a sum of three products.

This last result improves the main theorem in [W3], where factorisation was shown for finitely generated groups only and where the number of products depended on the number of generators of $G$ and the codimension of $I$.

It is possible to prove for non-discrete $G$ also that any ideal in $L^{1}(G)$ which has finite dimensional, commutative quotient factors. This can be done by using the above result for discrete groups and techniques from [W4]. However, a better proof would be one obtained by extending Theorem 3 to the non-discrete case.

Theorem 3 also provides an answer to a question of H. Reiter concerning closed, left ideals in group algebras. He asks, in [Re1], 8.4.6: if $I$ is a closed, left ideal in $L^{1}(G)$ and $N$ is a closed, normal subgroup of $G$, is the image of $I$ in the quotient algebra $L^{1}(G) / \operatorname{ker}\left(T_{N}\right)$ a closed ideal? (It is clear that this image is an ideal and so the point of the question is whether it is closed.) This is equivalent to the question as to whether the left ideal $I+\operatorname{ker}\left(T_{N}\right)$ is closed in $L^{1}(G)$.

In [Re1] Reiter showed that, in the case when $N$ is an amenable group, $I+\operatorname{ker}\left(T_{N}\right)$ is closed. However, examples show that the answer to Reiter's question, at least in the generality in which it was asked, is 'no'. Some examples given in [J] show that it is necessary to suppose that $N$ is amenable in order to obtain his result for all left ideals. Furthermore in [Ri] it is shown that, if $N$ is not amenable and has infinite index in $G$, then there are closed subspaces of $L^{1}(G)$, invariant under left translation by elements of $N$, whose image under $T_{N}$ is not closed. Here is another example showing that amenability is necessary.

Example. Let $\mathbb{F}_{2}$ be the free group with generators $a$ and $b$. Then the left ideal $\ell^{1}\left(\mathbb{F}_{2}\right) *\left(\delta_{e}+\delta_{a}+\delta_{b}\right)$ is closed in $\ell^{1}\left(\mathbb{F}_{2}\right)$; see [W5], Corollary 2.5. Now let $N$ be the commutator subgroup of $\mathbb{F}_{2}$. Then $\ell^{1}\left(\mathbb{F}_{2}\right) / \operatorname{ker}\left(T_{N}\right) \simeq \ell^{1}\left(\mathbb{F}_{2} / N\right) \simeq \ell^{1}\left(\mathbb{Z}^{2}\right)$. The image of $\ell^{1}\left(\mathbb{F}_{2}\right) *\left(\delta_{e}+\delta_{a}+\delta_{b}\right)$ in this commutative group algebra is a singly generated ideal which is easily seen to be not closed. 
If, however, $I$ is not just a left ideal but is two-sided, then Reiter's theorem does extend to the case when $N$ is not amenable.

Theorem 6. Let $N$ be a normal subgroup in the discrete group $G$, and let $I$ be a two-sided ideal in $\ell^{1}(G)$. Then $I+\operatorname{ker}\left(T_{N}\right)$ is a closed ideal in $\ell^{1}(G)$.

Proof. It suffices to show that, if $\left\{f_{n}\right\}_{n=1}^{\infty}$ is an absolutely summable sequence in $I+\operatorname{ker}\left(T_{N}\right)$, then its sum, $\sum_{n=1}^{\infty} f_{n}$, is also in $I+\operatorname{ker}\left(T_{N}\right)$.

For this, let $\mu_{1}$ and $\mu_{2}$ be probability measures such that

$$
\operatorname{ker}\left(T_{N}\right)={ }_{\mu_{1}} J+J_{\mu_{2}}
$$

and suppose that $f_{n}=h_{n}+k_{n}$, where $h_{n}$ is in $I$ and $k_{n}$ in $\operatorname{ker}\left(T_{N}\right)$. Then for each $n$ there are probability measures $\lambda_{1}^{(n)}$ and $\lambda_{2}^{(n)}$, convex combinations of powers of $\mu_{1}$ and $\mu_{2}$ respectively, such that $\left\|\lambda_{1}^{(n)} * k_{n} * \lambda_{2}^{(n)}\right\|<2^{-n}$, so that the sequence $\left\{\lambda_{1}^{(n)} * k_{n} * \lambda_{2}^{(n)}\right\}_{n=1}^{\infty}$ is absolutely summable. Since $\lambda_{1}^{(n)}$ and $\lambda_{2}^{(n)}$ are probability measures, the sequence $\left\{\lambda_{1}^{(n)} * f_{n} * \lambda_{2}^{(n)}\right\}_{n=1}^{\infty}$ is also absolutely summable. Hence the series $\sum_{n=1}^{\infty} \lambda_{1}^{(n)} * h_{n} * \lambda_{2}^{(n)}$ is absolutely convergent.

Let $h=\sum_{n=1}^{\infty} \lambda_{1}^{(n)} * h_{n} * \lambda_{2}^{(n)}$. Then, since $I$ is a closed ideal, $h$ belongs to $I$. The sequence $\left\{f_{n}-\lambda_{1}^{(n)} * h_{n} * \lambda_{2}^{(n)}\right\}_{n=1}^{\infty}$ is also absolutely summable and, furthermore, $f_{n}-\lambda_{1}^{(n)} * h_{n} \lambda_{2}^{(n)}$ belongs to $\operatorname{ker}\left(T_{N}\right)$ for each $n$. Hence, since $\operatorname{ker}\left(T_{N}\right)$ is closed, $k=\sum_{n=1}^{\infty} f_{n}-\lambda_{1}^{(n)} * h_{n} * \lambda_{2}^{(n)}$ belongs to $\operatorname{ker}\left(T_{N}\right)$. Therefore $\sum_{n=1}^{\infty} f_{n}=h+k$, which is in $I+\operatorname{ker}\left(T_{N}\right)$.

The argument used to deduce Theorem 6 from Theorem 3 and that used by Reiter are particular instances of a general theorem proved by Rudin; see [Ru], Theorem 4.2. Of course, a version of Theorem 6 for non-discrete groups would hold if Theorem 3 could be extended to cover the non-discrete case.

\section{REFERENCES}

[B-D] F. F. Bonsall and J. Duncan, Complete Normed Algebras, Springer-Verlag, Berlin, Heidelberg, New York, 1973. MR 54:11013

[C-D] G. Choquet and J. Deny, Sur l'équation de convolution $\mu=\mu * \sigma$, C. R. Acad. Sci. Paris Sér. Paris I Math. 250 (1960), 799-801. MR 22:9808

[D-S] N. Dunford and J. T. Schwartz, Linear Operators. Part I, Interscience, New York, 1957. MR 90g:47001a

[F] H. Furstenberg, A Poisson formula for semi-simple Lie groups, Ann. of Math. 77 (1963), 335-386. MR 26:3820

[H-R] E. Hewitt and K. A. Ross, Abstract harmonic analysis. I, Springer-Verlag, Berlin, Heidelberg, New York, 1963. MR 28:158

[J] B. E. Johnson, Some examples in harmonic analysis, Studia Math. 48 (1973), 181-188. MR 49:3456

[K] V. A. Kaimanovich, Bi-harmonic functions on groups, C. R. Acad. Sci. Paris Sér. I Math. 314 (1992), 259-264. MR 93e:60160

[M-Z] D. Montgomery and L. Zippin, Topological transformation groups, Interscience, New York, 1955. MR 17:383b

[Re1] H. Reiter, Classical harmonic analysis, Oxford University Press.

[Re2] H. Reiter, Sur certains idéaux dans $L^{1}(G)$, C. R. Acad. Sci. Paris Sér. A-B 267 (1968), A882-A885. MR 39:6025

[Ri] H. Rindler, Über ein Problem von Reiter und ein Problem von Derighetti zur Eigenschaft $P_{1}$ lokalkompakter Gruppen, Comment. Math. Helv. 48 (1973), 492-497. MR 49:1008

[Ru] W. Rudin, Spaces of type $H^{\infty}+C$, Ann. Inst. Fourier (Grenoble) 25, No. 1 (1975), 99-125. MR 57:7134; MR 51:13692 
[W1] G. A. Willis, Probability measures on groups and some related ideals in group algebras, J. Funct. Analysis 92 (1990), 202-263. MR 91i:43003

[W2] G. A. Willis, The structure of totally disconnected, locally compact groups, Math. Ann. 300 (1994), 341-363. MR 95j:22010

[W3] G. A. Willis, Factorization in codimension two ideals of group algebras, Proc. Amer. Math. Soc. 89 (1983), 95-100. MR 85c:43005

[W4] G. A. Willis, The continuity of derivations from group algebras: factorizable and connected groups, J. Austral. Math. Soc. 52 (1992), 185-204. MR 93b:46096

[W5] G. A. Willis, Translation invariant functionals on $L^{p}(G)$ when $G$ is not amenable, J. Austral. Math. Soc. 41 (1986), 237-250. MR 88b:43002

Department of Mathematics, The University of Newcastle, New South Wales, AusTRALIA, 2308

E-mail address: george@frey.newcastle.edu.au 